\title{
Privacy \& confidentiality: perspectives of mental health consumers \& carers in pharmacy settings
}

Hendrika Laetitia Hattingh ${ }^{a, b}$, Kathy Knox ${ }^{c}$, Jasmina Fejzic ${ }^{c}$, Denise McConnell ${ }^{b}$, Jane L. Fowler ${ }^{c}$, Amary Mey $^{b}$, Fiona Kelly ${ }^{c, d}$, Amanda J Wheeler ${ }^{c, d}$

\footnotetext{
${ }^{a}$ Curtin Health Innovation Research Institute, Curtin University, Bentley, Perth, Australia

${ }^{\mathrm{b}}$ School of Pharmacy, Griffith University Gold Coast, Parklands Drive, Southport, Gold Coast, Australia
}

${ }^{c}$ Griffith Health Institute, Griffith University, Brisbane, Australia

${ }^{d}$ Faculty of Medical and Health Sciences, University of Auckland, New Zealand

H Laetitia Hattingh (corresponding author)

Curtin Health Innovation Research Institute, Curtin University, Bentley, Perth, 6845, Australia

Griffith University Gold Coast, Parklands Drive, Southport, Gold Coast, 4226, Australia

Ph:+61892667376; Fax:+61892662769; email: I.hattingh@curtin.edu.au

Kathy Knox

Griffith Health Institute, Griffith University, Brisbane, 4131, Australia

Ph:+61733821242;fax:+61733821041 email: k.knox@griffith.edu.au

Jasmina Fejzic

Griffith University Gold Coast, Parklands Drive, Southport, Gold Coast, 4226, Australia

Ph:+61755529230; Fax:+61755528804; j.fejzic@griffith.edu.au

Denise McConnell

Griffith University Gold Coast, Parklands Drive, Southport, Gold Coast, 4226, Australia

Ph:+61733821241;fax:+61733821041 email:d.mcconnell@griffith.edu.au 
Jane L. Fowler

Griffith Health Institute, Griffith University, Brisbane, 4131, Australia

Ph:+61733821396; fax:+61733821210 ; email:j.fowler@griffith.edu.au

\section{Amary Mey}

Griffith University Gold Coast, Parklands Drive, Southport, Gold Coast, 4226, Australia

Ph:+61733821241;fax:+61733821041 email: a.mey@griffith.edu.au

Fiona Kelly

Griffith Health Institute, Griffith University, Brisbane, 4131, Australia

Faculty of Medical and Health Sciences, University of Auckland, New Zealand

Ph:+61733821496;fax:+61733821041 email: f.kelly@griffith.edu.au

Amanda J Wheeler

Griffith Health Institute, Griffith University, Brisbane, 4131, Australia

Faculty of Medical and Health Sciences, University of Auckland, New Zealand

Ph:+61733821068;fax:+61733821041 email:a.wheeler@griffith.edu.au

$\mathrm{LH}, \mathrm{JF}, \mathrm{AW}, \mathrm{JF}$ and $\mathrm{AW}$ devised the study and its methodology. KK and AM created the interview tools, sought ethics approval and were involved in the interviews. LH, KK, FK and AW did most of the data analysis. All authors had access to the data, contributed to all drafts of the paper and approved the final copy for publication.

Ethics approval was obtained from Griffith University's Human Research Ethics Committee (PHM/13/11/HREC) 


\section{ABSTRACT}

Objectives: To explore within the community pharmacy practice context the views of mental health stakeholders on 1) current and past experiences of privacy, confidentiality and support, and 2) expectations and needs in relation to privacy and confidentiality.

Methods: In-depth interviews and focus groups were conducted in three states in Australia, namely Queensland, the northern region of New South Wales, and Western Australia, between December 2011 and March 2012.

Key findings: There were 98 participants consisting of consumers and carers $(n=74)$, health professionals $(n=13)$ and representatives from consumer organisations $(n=11)$. Participants highlighted a need for improved staff awareness. Consumers indicated a desire to receive information in a way that respects their privacy and confidentiality, in an appropriate space. Areas identified that require improved protection of privacy and confidentiality during pharmacy interactions were the number of staff having access to sensitive information, workflow models causing information exposure, and pharmacies' layout not facilitating private discussions. Challenges experienced by carers created feelings of isolation which could impact on care.

Conclusions: This study explored mental health stakeholders' experiences and expectations regarding privacy and confidentiality in the Australian community pharmacy context. A need for better pharmacy staff training about the importance of privacy and confidentiality and strategies to enhance compliance with national pharmacy practice requirements were identified. Findings provided insight into privacy and confidentiality needs and will assist in the development of pharmacy staff training material to better support consumers with sensitive conditions.

\section{Keywords:}

privacy, confidentiality, pharmacy space, mental health 


\section{INTRODUCTION}

The Australian National Mental Health Consumer and Carer Forum (NMHCCF) has identified unique privacy and confidentiality needs of mental health consumers and carers as matters of concern. ${ }^{1}$ Mental health service staff members need to comply with privacy and confidentiality requirements as per the National Standards for Mental Health Services. ${ }^{2}$ Similarly, pharmacy staff require an understanding of the legal, professional and ethical obligations to maintain the confidentiality of mental health consumers' information and the mechanisms by which privacy of information is secured.

Pharmacy practice occurs in an environment where every health consumer expects their information will be kept private and confidential. ${ }^{3}$ However, strict adherence to privacy and confidentiality requirements in community pharmacy practice is complicated by the retail nature of community pharmacy and the need to provide personal health services in an open area. Pharmacy staff therefore need to implement strategies to overcome these privacy and confidentiality barriers. This is important as the management of privacy and confidentiality has an impact on relationships between consumers, carers and pharmacy staff ${ }^{4}$ which is more pronounced for vulnerable groups of healthcare consumers, such as mental health consumers. ${ }^{1}$

Mental illness is estimated to affect almost half of the Australian population at some stage in their lifetime and one in four people in any 12 -month period. ${ }^{5}$ Over recent years the management of consumers with mild to moderate depression or anxiety has shifted to primary health care providers at the community level. ${ }^{6}$ Community pharmacies are the primary suppliers of government subsidised medicines for these conditions..$^{7-9}$ Through the ongoing supply of prescription medicines community pharmacy staff members have regular contact with mental health consumers and carers and are in an ideal position to monitor adherence and progress, build trusting relationships and contribute to their medication-related needs. ${ }^{8-12}$

Australia's Fourth National Mental Health Plan emphasises that the rights and needs of consumers and carers must be recognised and monitored through efforts to improve their experience with mental health services. ${ }^{13}$ Most medicines used for mental illness require counselling about correct usage, potential adverse-effects, and ongoing monitoring ${ }^{14-16}$ and pharmacists should provide consumers with detailed information, advice and follow-up in a sensitive manner. However, people with mental illness are not unique, and any community pharmacy consumer with acute or chronic 
health care needs also requires detailed counselling about their medicines. ${ }^{17}$ Due to the sensitive nature of personal health information, conversations between pharmacists and consumers should take place in an area of the pharmacy that provides appropriate privacy. Pharmacy staff also need to be sensitive with regard to the way that dispensed medicines are stored and moved within the pharmacy to not disclose the name of the medicine and protect the identity of the person for whom they are intended. ${ }^{18}$ When followed, appropriate workflow processes and procedures facilitate privacy and confidentiality to ensure that customer information is used appropriately. ${ }^{4}$

The access to private and confidential information is based on the therapeutic relationship which exists between consumers of health services and health services staff. Privacy and confidentiality requirements are embedded in legislation, codes, standards and guidelines. ${ }^{19-21}$ The Pharmaceutical Society of Australia Code of Ethics recognises the obligation imposed upon pharmacists to ensure privacy and maintain confidentiality of consumer information. ${ }^{22}$ Although the Code specifically applies to pharmacists, other pharmacy staff members (pharmacy and dispensary assistants) are encouraged to comply with the principles and also need to be sensitive to the privacy and confidentiality requirements involved in the provision of pharmacy services. As pharmacists are ultimately accountable for services provided under their supervision it is important to ensure that all pharmacy staff members are trained in privacy and confidentiality requirements.

Research has indicated that private pharmacy areas in the United Kingdom were less accessible than intended, and utilisation of private consultation rooms posed a challenge as these areas were often used for other purposes or were not patient-friendly. ${ }^{23}$ An observational study regarding emergency hormonal contraceptive $(E H C)$ requests indicated that women felt less comfortable asking for the EHC in a pharmacy compared to a clinic because of privacy and confidentiality concerns. ${ }^{24}$ In an Australian study exploring the provision of EHC to a mystery shopper, four of 23 participating pharmacies used a private consultation area, seven offered a semi-private area, but other interactions took place in close proximity of pharmacy customers. ${ }^{25}$ Research with Australian healthcare consumers showed that lack of confidentiality and anonymity were perceived as barriers to using community pharmacy. ${ }^{26}$ Consumers with chronic conditions indicated that speaking to a pharmacist about medicine information was more likely if the layout of the pharmacy allowed privacy and if the dispensing area was separate from the front-of-shop. ${ }^{27}$ Stakeholder interviews and consumer forums identified that a lack of privacy, and at times lack of sensitivity, were shown to consumers by pharmacy staff and were main drawbacks to effective interaction. ${ }^{28}$ 
Internationally, large scale surveys of the general public have shown that more than half of respondents thought it was difficult to discuss intimate matters concerning medications ${ }^{29}$ or were reluctant to talk to a pharmacist about confidential issues ${ }^{30}$ due to the open nature of pharmacies. Pharmacy mystery shopper studies have highlighted a need for privacy to be acknowledged and respected. ${ }^{31}$ A Netherlands study reported that consumers experienced difficulties asking questions due to lack of privacy. ${ }^{32}$ The apparent lack of privacy can impact consumer behaviour and pharmacy choice: systematic reviews show that pharmacy users expressed concerns about the level of privacy, and selected or deselected a community pharmacy depending on facilities for private discussion. ${ }^{3}$ However, there is some evidence that pharmacy staff exhibit behaviour perceived as appropriate by consumers such as speaking quietly and utilising a private area within the pharmacy. ${ }^{33}$

Recent Australian research indicates that people with mental illness often experience stigma and discrimination, especially in media portrayals. ${ }^{34}$ A 2011 report identified that $73 \%$ of people surveyed ( $>400$ adults) had experienced stigma or discrimination in the previous 12 months because of their mental illness. ${ }^{34}$ That figure was no different to a similar 2006 survey. ${ }^{35}$ Given that mental health consumers can be sensitive to both self-stigma and public stigma in the community pharmacy context $^{36}$, it is particularly important that their privacy and confidentiality needs are managed in a sensitive way. Pharmacy staff should recognise the needs of mental health consumers and provide the appropriate level of privacy whilst not making them feel as though they are being singled out. However, in-depth research considering mental health consumers' needs, experiences and perceptions of privacy and confidentiality in the Australian community pharmacy setting is lacking. A need was therefore identified to explore the unique privacy and confidentiality requirements of mental health consumers and carers in the Australian community pharmacy context.

\section{METHODS}

This study was conducted in three states in Australia (Queensland, New South Wales, and Western Australia) between December 2011 and March 2012. These jurisdictions were chosen as they included urban, regional, rural and remote, culturally diverse and indigenous populations to ensure that the data collected represented a wide range of community settings. Data were collected using qualitative methodology to allow for an in-depth exploration of stakeholders' views. Ethics approval was obtained from the relevant University's Human Research Ethics Committee. Participants received a small gift as a token of appreciation. 
In-depth consultation with mental health consumers and carers, health care professionals, and representatives from consumer/carer organisations were undertaken to explore, within the community pharmacy context:

1. current and past experiences of privacy, confidentiality and support, and

2. expectations and needs in relation to privacy and confidentiality.

\section{Recruitment and Data Collection}

Both purposive and convenience sampling methods were used to recruit interview participants. Potential participants were classified into three categories according to their experiences and roles: health professionals, mental health consumer/carer organisational representatives and mental health consumers/carers.

To select the health professional and organisation representatives, an initial sampling framework of key informants working in relevant roles in the three states was developed in consultation with the project's reference group. The health care professionals and representatives from mental health consumer/carer organisations were purposively sampled to reflect diversity across the consumer support organisations, pharmacy and health professional sectors. To achieve data saturation, 18 health care professionals and 27 consumer/carer organisation representatives were approached to participate. Consumer and carer participants were a convenience sample supplemented by snowballing (participants identified other suitable participants) invited to participate in a focus group discussion regarding the role of community pharmacy in mental health care, which was advertised and promoted on behalf of the researchers by the participating consumer/carer organisation representatives.

Those interested to be interviewed received an information sheet about the study that included contact details of the researchers and an invitation to ask further questions about the study and the data collection and analysis processes. All participants signed a consent form. Participating health care professionals and consumer/carer organisation representatives were interviewed individually at a time and place that was convenient for the participant. Four interviews were face-to-face and the remainder were conducted over the telephone. Each interview was approximately 30 minutes in duration (range 11 to 45 minutes). Focus group interviews took place in conference rooms at community mental health centres. Two trained facilitators were present for each focus group with the same facilitator taking the lead in all focus group interviews to ensure consistency, while the 
other facilitator took field notes. Focus group interviews were on average 1 hour in duration (range 40 minutes to 1 h. 40 minutes).

Interview guides allowed for the collection of qualitative narrative data through dialogue between the interviewer and participant(s). Interviews took the form of a conversation using open-ended trigger/seed questions. Separate guides were developed for use with consumers and carers, or with health professionals and consumer/carer organisation representatives (Table 1). Focus group discussions were guided by similar prompts as were used in individual interviews, with the inclusion of introductions and opening statements, ground rules and warm-up questions.

$<$ Table $1>$

\section{Data Analysis}

The transcribed, de-identified qualitative data was prepared for analysis by conducting quality checks on a sample of interviews (i.e., a researcher not involved in conducting or transcribing the interview listened to the recording whilst reading over the transcript in order to check for accuracy). Data collection ceased when data saturation was achieved.

A software package (NVivo ${ }^{\circledR}$ Version 9.0) was used to organise the data. Thematic analysis of the data was informed by the general inductive approach ${ }^{37}$ and involved multiple stages. First, transcripts were read and re-read by members of the research team to gain an understanding of the broad issues relative to the key evaluation questions. Then, specific themes were developed which captured core messages reported by the participants. To ensure the process was reliable, two researchers coded and grouped data into thematic categories. An additional consistency check was conducted on a sample of transcripts to verify that data was coded in a similar way by a third research team member who had not been involved in conducting, transcribing, or coding of the interviews. The multiple-stage analysis process was undertaken to code 'units' of data, then categorise ('cluster') the units until themes emerged.

\section{RESULTS}

Participants were 98 adults: health professionals $(n=13)$, mental health consumer/carer organisational representatives $(n=11)$, and mental health consumers/carers $(n=74)$. The 
professionals interviewed were seven male and six female health care professionals working in a range of positions including a psychologist, general practitioner, psychiatry registrar, mental health nurse, mental health pharmacist, and registered nurse. Mental health consumer/carer organisation respondents who were interviewed were eight females and three males working in roles such as consumer consultant, carer consultant, mental health manager or worker, support worker, or social worker. The consumers/carers were 26 males and 48 females. Nine consumer/carer focus group interviews were conducted ( $n=66$ ). Eight consumers/carers elected to give an individual interview; three were conducted face-to-face, and five over the telephone. Sixty focus group participants provided information about their age and self-reported their ongoing mental health conditions (Table 2). Some participants reported that they had experienced more than one mental health condition.

$<$ Table $2>$

Multiple privacy and confidentiality themes emerged from the interview data. Quotes were coded to indicate whether the participant was a consumer (C), carer (CR) or both (C/CR) and the codes FG and SSI indicate whether the information was gathered in a focus group setting or a semi-structured interview. Health professionals are denoted as HP, and consumer/carer organisation representatives as OR.

The main privacy and confidentiality themes identified involved recognising pharmacy as a public space, use of private consultation areas, the role of pharmacy staff, specific carer needs and strategies to facilitate privacy and confidentiality. These themes are discussed using examples from the transcripts to illustrate the dimensions of each theme.

\section{Pharmacy is a Public Space}

Consumers and carers expressed concerns that their anonymity and right to receiving sensitive information were breached when other customers were present in the pharmacy. For example, other customers in the pharmacy could see them, hear their name and address being called out (customers' names are routinely used for verification when collecting prescriptions), might see or hear which medications they were receiving, and overhear them being counselled. Due to the highly accessible nature of community pharmacy services and services being provided in a public space, there is a fear of being recognised by colleagues, friends and neighbours when collecting medication. 
These fears are particularly heightened in smaller communities where there is also a greater chance of a personal association with pharmacy staff. Some consumers chose to access pharmacies at a greater distance to avoid this:

"I went to a different pharmacy so that no-one would recognise me. Because I have lived in the area 20 years at the time and I thought, 'I cannot do this. I cannot stand there with [antidepressant] or [stimulant medicine] while I pay in case somebody comes in and I found that incredibly intrusive into my personal self."' (C/CR SSI 120206)

"[Pharmacy] can be perceived by the consumer as quite a public space. Perhaps the pharmacist has a more public conversation where other people can overhear it. Some consumers are very sensitive to what information might be discussed." (HP02)

\section{Private Consultation Area}

The use of a private consultation room or area was seen as a main facilitator for overcoming privacy and confidentiality issues during pharmacy interactions:

"I think the chemist should all have a section that you can talk privately regardless. Because you might want to ask something personal and not to do with medication. I think there should be a section anyway. Whether you want get something from the nurse, the pharmacist, the pharmacy assistant or the shop keeper. Yeah, or you know, for anybody, for any reason. If there is a section there where the pharmacist, you can talk privately there about something." (C FG111207)

Interestingly, making consultations with mental health consumers 'more confidential' (i.e. taking them to a privateconsultation room) rather than managing them the same way as other consumers was perceived as both desirable and undesirable. One consumer specifically reported that it made the consumer feel like he/she was being singled out, which can propagate the existing stigma associated with mental illness:

"Some community pharmacies do have a patient consult area but they are often quite small rooms. Sometimes, being taken away to a small room makes the individual uncomfortable, perhaps, because he is being taken down to the back ... to have a more private conversation." (HP02) 


\section{Role of Pharmacy Staff}

Some consumers expressed concerns regarding all pharmacy staff knowing about their medical history through employment of multiple pharmacists, pharmacy support staff over-hearing or being involved in the conversation, and the pharmacy dispensing process where one support staff member takes in the prescription and another staff member hands the medication to the consumer:

"It's not just the pharmacists and that is, what, four or five of them on different shifts, but also the assistant behind the counter as well." (C FG 111207)

"It just seems really impersonal ... I'm getting some serious medications ... you go up and you tell one person what you are getting then someone else will come back and say 'Here is your medication,' then you go to someone else to pay for it. It just seems really like everyone knows your business there. I don't like that." (C SSI 120307)

There was also consumers' fear of being recognised by a pharmacy staff member who knows the consumers in a private capacity when collecting medication from the pharmacy and being judged for having a mental illness, "My friend's two girls work behind the counter. I know they are discreet ... but it's just that my perception is [I'm] being judged." (C/CR SSI 120206)

\section{Carer Needs}

An issue particular to carers was the difficulty encountered due to privacy laws when accessing information. Carers, who are oftensupporting a family member, do experience obstacles in communicating timely and accurate information, "Carers, even though we have come a long way, still tend to be kept outside of the information loop." (OR07)

One example of acceptable practice in this regard was communicated by a consumer /carer:

“... a situation where I had to sort out my son's prescription over the phone and then go back to the pharmacy to get the fax number and explain to them that we were on holiday and he has forgotten his antipsychotic medication and they will be faxing through his script here. They were very patient and helpful and that made it less stressful because my son was having a psychotic episode having not taken his medications ... my expectation of the pharmacy is to be knowledgeable and to go the extra mile to be helpful and understanding." (C/CR SSI 120208) 
The anecdotal evidence emerging from this study illustrates carers' predicament and the potential role that community pharmacy can have in managing sensitive information appropriately. From the carer's perspective, there was a reported requirement to balance therapeutic needs with privacy constraints:

" $[\mathrm{A}]$ decision has to be made regarding patient privacy versus best care for the patient, go that extra mile to secure continuation of therapy, listen to the carer. They have another perspective of the same story and can give you [pharmacy staff] very important information." (C/CR SSI 120206)

\section{Proposed Strategies for Improvement}

Consumers/carers provided a range of strategies to address the lack of pharmacy privacy, which reflected a lateral approach to addressing the limitations imposed by the pharmacy layout and workflow. A number of strategies revealed a consumer desire for person-centred, respectful care from pharmacists and support staff, as summarised in Table 3.

$<$ Table $3>$

Although the majority of respondents expressed a desire for their privacy and confidentiality to be protected, it was also generally acknowledged that pharmacy staff were doing their best under the circumstances, but that consumers' general privacy, and expectation of privacy, had nevertheless, also been somewhat eroded in other public spaces:

"You can walk into so many places nowadays, Centrelink [Australian Government organisation that delivers social and health-related payments and services] or Immigration or whatever, and you are talking your private lives next to people sitting next to you." (C FG120224)

\section{DISCUSSION}

This exploratory study provided insight into the perspectives of mental health stakeholders regarding their privacy and confidentiality needs and expectations of community pharmacies. Stakeholders expressed a need for improved protection of privacy and confidentiality during community pharmacy interactions as part of providing a welcoming, safe, and trusting healthcare environment. Although stakeholders provided instances of appropriate privacy and confidentiality 
practices, the lack of private areas and multiple pharmacy staff having access to sensitive information were main concerns. The need to improve workflow models and processes to reduce consumer exposure during dispensing and counselling also emerged. Consumers indicated a desire to receive information in a way that respects their privacy and confidentiality, in an appropriate space. Carers identified specific practices that could hinder patient care.

This study had certain limitations namely relying on convenience sampling of consumers and carers in three states with some snow-balling. However, calling for volunteers to participate in the research via consumer and carer organisations and networks was deemed an appropriate method to approach this vulnerable population. ${ }^{38}$ Self-reported data (in-depth interviews) was used which can be influenced by social desirability response and interviewer bias. However, the potential for interviewer bias was minimised with the use of a standardised interview framework. As a further means of assuring analytic rigour, steps were taken to guard against selectivity in the use of data such as employing multiple coders and analytic process that sought to ensure inter-coder consistency. Purposive sampling was used to locate key stakeholders, limiting the generalizability of the findings. However, purposive sampling was deemed the most appropriate method of ensuring that the perspectives of stakeholders were included. ${ }^{38}$

Participants identified a need for a separate area to be used by staff during interactions with any customers where personal or confidential information is being discussed, including mental health concerns. They expressed concern over the relative lack of privacy within community pharmacies and the potential for acquaintances to be in this space at the same time, particularly in smaller communities. Although other health care settings also have public waiting areas this was identified as a particular issue with regard to pharmacy practice, especially if sensitive issues were involved and conversations were not carried out in a private manner. The findings support previous research regarding the lack of pharmacy privacy, ${ }^{16,39,40}$ and reiterate that interventions such as changing pharmacy layouts to create a professional services area using barriers or creating an area away from pharmacy traffic are warranted. ${ }^{24,41}$ Participants also identified simple strategies to improve privacy and confidentiality such as staff moving around the counter to stand next to the consumer. Such solutions are easy to implement and address practice challenges posed by space.

A busy pharmacy imposes a number of conflicting demands on staff behaviour and attention and staff might lapse in their consideration of a consumer's privacy and confidentiality needs when managing waiting workloads. Strict adherence to privacy guidelines in community pharmacy is 
further complicated by the retail element and requires careful planning. Although compliance with privacy requirements could be challenging pharmacy managers should pay attention to the pharmacy layout to facilitate privacy requirements. Although strategies to address some of these barriers may require financial investment, community pharmacies are already required to have a screened area or separate room that is distinct from the general public area of the pharmacy to qualify for participation in the recently introduced Australian government funded medication management services. ${ }^{42}$

A number of alternative strategies could facilitate communication between pharmacy staff and consumers and carers. These include telephone calls which could be organised to take place during a time convenient to both the pharmacist and the consumer, with both parties having more control in choosing where the conversation takes place. A novel recommendation stemming from the current findings is that pharmacy staff explore consumers' preferences for communication and implement the use of technology such as emails or text messages, for prescription reminders.

Doubts were expressed over confidentiality of consumers' medical information and were linked to pharmacy processes requiring multiple staff members to handle prescription(s), and to consumer assumptions about the professionalism of support staff. The therapeutic partnership between pharmacists and their customers is recognised by the Pharmacy Board of Australia as requiring "high standards of personal conduct... [which involve] protecting the privacy and right to confidentiality of patients and clients, unless release of information is required by law or by public interest considerations". ${ }^{432}$ These requirements extend to pharmacy support staff who should understand the legal, professional and ethical obligations to maintain the confidentiality of client information and the mechanisms by which privacy of, and access to, such information is secured.

Carers experienced challenges, feelings of isolation and a perceived a lack of recognition of situations when sharing information about the consumer might be necessary and justifiable; a finding similar to issues raised by the NMHCCF. ${ }^{1}$ Carers who are unrelated to the consumer reported difficulties in accessing consumer health information because of privacy laws. The difficulties that carers face may reflect knowledge gaps with the application and understanding of the privacy laws in practice, and signifies a need for targeted education for community pharmacy staff that will address carers' needs. Additional training of pharmacy staff in regards to information sharing with carers seems justified. 


\section{CONCLUSIONS}

Privacy and confidentiality requirements in community pharmacy involve the physical environment and chosen location within the pharmacy utilised by pharmacy staff for history taking and counselling, and the manner in which pharmacy staff deal with consumers to facilitate a trusting environment. Obtaining the perspective of people with mental illness in terms of their experiences, needs and expectations indicated that Australian community pharmacy staff may not be sufficiently prepared for managing consumer privacy and confidentiality, particularly in the context of mental health. This study identified an overall need for better staff training about the importance of privacy and confidentiality and how the pharmacy environment and workflow can influence this. These findings provided valuable insight into consumer and carer privacy and confidentiality needs and will assist in the development of more innovative and better practices, training packages and mentoring programmes to enable community pharmacy staff to consider the potential impact of the pharmacy environment to better support healthcare consumers and improve health outcomes.

\section{Acknowledgements}

The authors acknowledge the members of the Reference Group for their guidance and support and all the consumers, carers, representatives from mental health consumer and carer organisations, and health professionals who participated in this study.

This project was funded by the Australian Government Department of Health and Ageing as part of the Fifth Community Pharmacy Agreement Research and Development Program managed by the Pharmacy Guild of Australia. 


\section{References}

1. National Mental Health Consumer \& Carer Forum (NMHCCF). Privacy, confidentiality \& information sharing - consumers, carers \& clinicians2011. Available from: http://www.nmhccf.org.au/documents/NMHCCF\%20P\&C\%20ps\%20\&\%20ip.pdf.

2. National Mental Health Working Group. National standards for mental health services. Canberra, ACT: Australian Governement Publishing Serivce; 1996.

3. Anderson C, Blenkinsopp A, Armstrong M. Feedback from community pharmacy users on the contribution of community pharmacy to improving the public's health: a systematic review of the peer reviewed and non-peer reviewed literature 1990-2002. Health Expectations. 2004;7(3):191202.

4. Low J, Hattingh H, Forrester K. Australian Pharmacy Law and Practice. p 148. Sydney: Elsevier; 2010.

5. Australian Institute of Health and Welfare. Australia's Health 2012. Canberra: AlHW; 2012 [cited $2012 \quad 13 \quad$ August 2012]; Available from: http://www.aihw.gov.au/WorkArea/DownloadAsset.aspx?id=10737422169.

6. Slade T, Johnston A, Oakley Browne MA, Andrews G, Whiteford H. 2007 National Survey of Mental Health and Wellbeing: methods and key findings. Australian and New Zealand Journal of Psychiatry. 2009;43(7):594-605.

7. Australian Government Department of Health and Ageing. PBS and your prescription medicines2012. Available from: http://www.humanservices.gov.au/customer/subjects/pbs-andyour-prescription-medicines.

8. Australian Institute of Health and Welfare. Australia's health 2010: in brief2010. Available from: http://www.aihw.gov.au/publication-detail/?id=6442468375.

9. Australian Institute of Health and Welfare. Mental health services in Australia 2007-20082010. Available from: http://www.aihw.gov.au/publication-detail/?id=6442468381\&tab=2.

10. Wilson J. Mental health services in New Zealand. International Journal of Law and Psychiatry. 2000;23(3-4):215-28.

11. World Health Organization and World Organization of Family Doctors (Wonca). Integrating mental health into primary care: a global perspective2008. Available from: http://whqlibdoc.who.int/publications/2008/9789241563680_eng.pdf.

12. Cooper RJ, Bissell P, Wingfield J. 'Islands' and 'doctor's tool': the ethical significance of isolation and subordination in UK community pharmacy. Health:. 2009;13(3):297-316.

13. Commonwealth of Australia. Fourth National Mental Health Plan: An agenda for collaborative government action in mental health 2009-2014. Canberra: Commonwealth of Australia; 2009.

14. Sleath B, Wurst K, Lowery T. Drug information sources and antidepressant adherence. Community Mental Health Journal. 2003;39(4):359-68.

15. Happell B, Manias E, Roper C. Wanting to be heard: mental health consumers' experiences of information about medication. International Journal of Mental Health Nursing. 2004;13(4):242-8.

16. Phokeo V, Sproule B, Raman-Wilms L. Community pharmacists' attitudes toward and professional interactions with users of psychiatric medication. Psychiatric Services. 2004;55(12):1434-6.

17.Pharmaceutical Society of Australia. Professional Practice Standards. 4th edition ed. Canberra: Pharmaceutical Society of Australia; 2010.

18.Pharmacy Board of Queensland. Guidelines on Pharmacy and Confidentiality. Brisbane: Pharmacy Board of Queensland; 2007.

19.Skene L. Law and Medical Practice-Rights, Duties, Claims and Defences 3rd edn ed. Sydney: Lexis Nexis Butterworths; 2008.

20.LinkBG, Phelan JC, Bresnahan M, Stueve A, Pescosolido BA. Public conceptions of mental illness: labels, causes, dangerousness, and social distance. American Journal of Public Health. 1999;89(9):1328-33. 
21.Australian Government. Commonwealth Privacy Act. 1988; Available from: http://www.comlaw.gov.au/Series/C2004A03712.

22.Pharmaceutical Society of Australia. Code of Ethics for Pharmacists: Pharmaceutical Society of Australia; 2011. 1-11 p.

23. Rapport F, Doel MA, Jerzembek GS. "Convenient space" or "a tight squeeze": Insider views on the community pharmacy. Health and Place. 2009;15(1):315-22.

24.Black KI, Mercer $\mathrm{CH}$, Kubba A, Wellings K. Provision of emergency contraception: a pilot study comparing access through pharmacies and clinical settings. Contraception. 2008;77(3):181-5.

25. Higgins $\mathrm{S}$, Hattingh $\mathrm{H}$. Requests for emergency contraception in community pharmacy: An evaluation of services provided to mystery patients. Research in Social and Administrative Pharmacy. 2012; In press.

26. Gilbert A, Vawser L, Australia. Rural Health Support E, Program T, Pharmacy UoSASo, Sciences M, et al. Pharmacy Rural Health Project Report May 1996: Report of a Project Optimising the Health Impact of Rural and Remote Pharmacists on the Communities They Serve1996.

27.Consumers' Health Forum of Australia. Pharmaceuticals project - Final Report. Lyons, ACT: Department of Human Services and Health 1995.

28. Dunphy D, Palmer I, Benrimoj SL, Roberts A. The Final Report of the Change Management and Community Pharmacy Project. Canberra: Pharmacy Guild of Australia; 2005.

29. Airaksinen M, Ahonen R, Enlund H. The "Questions to Ask about Your Medicines" Campaign: An Evaluation of Pharmacists and the Public's Response. Medical Care. 1998;36(3):422-7.

30. Bell H, McElnay J, Hughes C. Societal perspectives on the role of the community pharmacist and community based pharmaceutical services. Journal of Social and Administrative Pharmacy. 2000;17(2):119-28.

31. Norris $\mathrm{P}$, Rowsell B. Interactional issues in the provision of counselling to pharmacy customers. International Journal of Pharmacy Practice. 2003;11(3):135-42.

32. Pronk MCM, Blom ATG, Jonkers R, Bakker A. Evaluation of patient opinions in a pharmacy-level intervention study. International Journal of Pharmacy Practice. 2003;11(3):143-51.

33. Bawazir SA. Consumer attitudes towards community pharmacy services in Saudi Arabia. International Journal of Pharmacy Practice. 2004;12(2):83-9.

34.SANE Media Centre. Stigma hurtful and common for people with mental illness. 2011.

35.SANE Media Centre. Stigma of mental illness means people reluctant to seek treatment. 2007

36. Black E, Murphy AL, Gardner DM. Community pharmacist services for people with mental illnesses: preferences, satisfaction, and stigma. Psychiatr Serv. 2009;60(8):1123-7.

37. Thomas DR. A general inductive approach for analyzing qualitative evaluation data. American Journal of Evaluation. 2006;27(2):237-46.

38. Buetow S. Health Research Methods: a Tabular Presentation. South Carolina, U.S.A: Nova Biomedical Books; 2007. 168 p.

39. Cates ME, Burton AR, Woolley TW. Attitudes of pharmacists toward mental illness and providing pharmaceutical care to the mentally ill. Annals of Pharmacotherapy. 2005;39(9):1450-5.

40.Scheerder G, De Coster I, Van Audenhove C. Pharmacists' role in depression care: a surveyof attitudes, current practices, and barriers. Psychiatric Services. 2008;59(10):1155-60.

41. World Health Organization. The world health report 2001: Mental health: New understanding, new hope. Geneva, Switzerland: World Health Organization; 2001 [cited 201106 April]; Available from: http://www.who.int/whr/2001/en/whr01_en.pdf.

42. Department of Health and Ageing, The Pharmacy Guild of Australia. Program Specific Guidelines MedsCheck and Diabetes MedsCheck. Canberra: Department of Health and Ageing; 2012.

43.Pharmacy Board of Australia. Pharmacy Code of Conduct for Registered Health Practitioners2012. Available from: http://www.pharmacyboard.gov.au/Codes-Guidelines.aspx. 


\section{Consumer/Carer}

Health Professional/

\section{Organisation Representative}

\author{
Current level of knowledge of community \\ pharmacy \\ Expertise of the pharmacist \\ Role of the pharmacist, of support staff \\ Services provided / available \\ Current needs from community pharmacy \\ Deficiencies in service currently provided \\ Who is responsible for changes \\ Engagement in changes
}

Past and present experiences of community pharmacy

Current use of community pharmacy in management of mental illness

Strengths of current experience

Who is responsible for changes

Perceptions of medicine taking

Role of community pharmacy / consumer

Concerns

Barriers to adherence /motivation

Efficacy of treatment management Potential

future role for pharmacy

Cultural needs/beliefs/issues
Demographics and professional role

Experience with mental health consumers/carers

Policies and protocols

Current concerns regarding management of mental illness

Consumer/ carers needs from community pharmacy

Current use of pharmacy

Current problems or concerns from

consumers/carers

Impact of treatment burden

Potential future role of community pharmacy Role of community pharmacy in management of mental health

Improving adherence

Gaps, barriers or opportunities

Cultural needs/beliefs/issues

Questions/comments/other views to share 
Table 2. Consumer/ carer participant characteristics

\begin{tabular}{llc}
\hline Age group & & $\boldsymbol{n}$ \\
\hline & $18-25$ years & 3 \\
& $26-40$ years & 13 \\
& $41-55$ years & 23 \\
Mental health condition & 56 years + & 22 \\
& & \\
& Depression & 23 \\
& Anxiety & 18 \\
& Bipolar affective disorder & 11 \\
& Schizophrenia or other psychotic disorder & 13 \\
\hline
\end{tabular}


Table 3. Strategies to improve privacy and confidentiality

\begin{tabular}{ll}
\hline \multicolumn{1}{c}{ Strategy } & \multicolumn{1}{c}{ Example } \\
\hline $\begin{array}{l}\text { Dispensing medications in plain } \\
\text { packaging }\end{array}$ & "My pharmacy has taken to dispensing psychiatric \\
& medications in the plain white boxes which I find is really \\
& good. Providing it is well labelled, because of course only \\
I know I collect three white boxes once a fortnight." (C FG \\
111207)
\end{tabular}

Staff come out from behind the counter and guide the consumer to a separate area

Staff to be more discrete when asking questions, giving medicines advice and counselling
"Not everyone will take you up on it. Some people are quite happy discussing their medications in very loud voices in front of everybody, but it is giving the option, the choice and this is what we need; it is option and choices about the service that we are getting delivered." (C FG 120206)

"... the whole model that I am presenting, which is about sanctuary and respect, means that privacy would be fundamental to that." (OR11)

"What does bother me is if I say 'Can I have some Panadol ${ }^{\circledR \prime}$ over the counter and they say 'Are you on any other medication?' And I say ' $x$ and $x$ ' and they go 'Oh what's that for is it for epilepsy? What's that for?' And it is like that is when I stop then I say, 'I know it's OK for me to take that because I have checked before.' That's when I feel anxiety because you have got people standing around and it is like 'Oh that is for a mood disorder' so that is where I think there needs to be a little more discreetness." (C FG 120224) 